EPJ manuscript No.

(will be inserted by the editor)

\title{
A novel simplified two-dimensional magneto-optical trap as an intense source of slow cesium atoms
}

\author{
N. Castagna, J. Guéna a , M.D. Plimmer and P. Thomann
}

Observatoire cantonal, rue de l'Observatoire 58, CH-2000 Neuchâtel, Switzerland, e-mail: Natascia.Castagna@ne.ch

Received: date / Revised version: date

\begin{abstract}
We describe the design and performance of a slow atom source based on a 2D magneto-optical trap (MOT) that uses an innovative simple optical configuration. Metal-coated retro-reflecting prisms replace mirrors and quarter-wave plates so the optical power of the cooling laser beam is recycled. This source has been characterised for three different configurations: with and without transverse magnetic field gradient, and with a pusher beam to obtain a $2 \mathrm{D}^{+}$-MOT. The longitudinal velocity is of the order of $25 \mathrm{~m} \cdot \mathrm{s}^{-1}$, with a transverse velocity spread $\leq 1 \mathrm{~m} \cdot \mathrm{s}^{-1}$, while the typical atomic flux density obtained is up to $1.3 \times 10^{14} \mathrm{at} \cdot \mathrm{s}^{-1} \cdot \mathrm{m}^{-2}$ for a cesium vapour pressure of $\sim 4 \times 10^{-8} \mathrm{mbar}$ in the source. We use this slow atom beam, instead of cesium vapour, to load a 3D moving optical molasses that feeds a continuous cold atom fountain. We obtain a gain of a factor $\sim 20$ in the atomic flux launched by the fountain.
\end{abstract}

PACS. 06.30.Ft Time and frequency - 32.80.Lg Mechanical effects of light on atoms, molecules, and ions 32.80.Pj Optical cooling of atoms; trapping - 39.10.+j Atomic and molecular beam sources and techniques - 39.25.+k Atom manipulation (scanning probe microscopy, laser cooling, etc.)

\section{Introduction}

Slow atomic beam sources represent a useful tool for many applications: atom interferometers [1-3], tests of fundamental symmetries [4,5], atomic clocks [6,7], Bose-Einstein

a On leave from Laboratoire Kastler Brossel (UMR 8552 CNRS), 24 rue Lhomond, 75005 Paris, France. condensation [8] and atomic and molecular spectroscopy [9]. The work described here is motivated by the development of a primary time standard using a continuous atomic fountain [10]. Such fountains have the advantage of a lower atomic density for a given signal-to-noise ratio than their pulsed counterparts, which helps minimise density related frequency shifts [6]. Furthermore, they enable 
one to eliminate the intermodulation (a.k.a. Dick) effect associated with local oscillator noise [11]. In order to take full advantage of the continuous fountain approach however, we need to increase the useful flux. One way is to launch more atoms to begin with. This can be achieved by loading the moving optical molasses source from a slow beam rather than by capture from the low-velocity tail of a thermal vapour.

Slow beam sources can be produced in various ways [1217]. Zeeman slowing of an atomic beam [14] allows a continuous source but is somewhat cumbersome and requires strong magnetic fields, while chirp slowing [18] provides only packets of atoms. Slow beams with low longitudinal velocity can be obtained by drilling a hole in one of the mirrors in a 3D-magneto-optical trap (3D-MOT) $[19,20]$ or in a pyramidal trap $[12,15]$. In $[21]$ the authors slow a thermal beam by using a counter-propagating laser beam and trap the atoms in a 3D-optical molasses superimposed to a 2D-magnetic field gradient. These techniques require a laser beam to be superimposed with the atomic beam. Slow beams can also be extracted from a static 3D-optical molasses superimposed to a 2D-magnetic field gradient using a static magnetic field along the beam axis [22]. Here we report on a much simpler solution, namely a $2 \mathrm{D}$ magneto-optical trap of cesium atoms producing a continuous low velocity beam $\left(v \simeq 25 \mathrm{~m} \cdot \mathrm{s}^{-1}\right)$. While 2D-MOT's have already been employed elsewhere [3,19,22-25], the devices in question are usually somewhat expensive, requiring many large $(\sim 30 \mathrm{~mm})$ anti-reflection coated polarising beam splitter cubes, quarter- and half-wave plates.
Furthermore, the laser power is divided at each stage so several hundred milliwatts are often necessary at the input. In our version, gold-coated prisms replace mirrors and quarter-wave plates and the optical power $(<200 \mathrm{~mW})$ is recycled. The net result is a considerable saving both in complexity and financial cost without degrading performance as regards the useful flux extracted from the source. In this article we describe the characterisation of the slow beam (longitudinal and transverse velocity distribution, spatial profile) and the optimisation of various parameters (laser power and polarisation, magnetic field gradient henceforth $\nabla \mathrm{B}$, laser detuning, cesium vapour pressure). We compare the results obtained with a magnetic gradient (2D-MOT), without one, i.e. the so-called 2D optical molasses (2D-OM), and in a third configuration where a pusher laser co-propagates with the atomic beam $\left(2 \mathrm{D}^{+}\right.$MOT).

The paper is organised as follows. Section 2 describes the experimental set-up. In Section 3 we present the measurement techniques and diagnostics of the atomic beam emerging both from the 2D-MOT and 2D-OM configurations. A comparison with other beam sources is given in Sec. 4. In Section 5 we compare the capture efficiency in a $3 \mathrm{D}$ static optical molasses fed by the slow atomic beam with that obtained using thermal vapour loading. In Section 6 we treat the situation where the atomic beam captured in the molasses is launched vertically. We analyse the upward flux from the optical molasses loaded from the 2D-MOT and 2D+-MOT beam sources and compare results with thermal vapour loading. This study is impor- 
tant for evaluating the potential improvement in stability achievable for an atomic fountain clock [10] using such a pre-source.

\section{Experimental set-up}

An overview of the set-up is given in Fig. 1. The 2DMOT pre-source to be described in Sec. 2.1 provides a slow atomic beam directed towards a 3D-optical molasses region (described in Sec. 2.2) where it is captured and then launched vertically in a cold atom fountain. We define two coordinate systems, $\{x, y, z\}$ and $\left\{x^{\prime}, y^{\prime}, z^{\prime}\right\}$ related to the laboratory and to the pre-source respectively. $x$ and $y$ represent the horizontal plane and $z$ the vertical launch direction of the fountain. $z^{\prime}$ designates the cell axis, inclined at $45^{\circ}$ to the vertical, and $x^{\prime}$ and $y^{\prime}=y$ lie in the transverse plane. All laser beams are transmitted from the same optical table to the slow atom beam apparatus through polarisation-maintaining optical fibres. Collimators at the fibre exits allow adjustment of beam diameters.

\subsection{Pre-source}

A scheme of the pre-source is represented in Fig. 2. The glass cell (internal dimensions $30 \mathrm{~mm} \times 30 \mathrm{~mm} \times 150 \mathrm{~mm}$ ) is connected to a UHV chamber via a titanium adaptor flange of internal diameter $16 \mathrm{~mm}$ that widens to $40 \mathrm{~mm}$ towards the vacuum chamber. The former diameter constitutes the smallest constriction between the pre-source and the 3D-OM region (Fig. 1), centre of the UHV chamber $500 \mathrm{~mm}$ downstream. Cs is injected directly into the pre-source cell. An adjustable bellows allows angular adjustment of cell axis while a gate valve enables one to isolate the pre-source from the vacuum system if necessary. The required $\nabla \mathrm{B}$ is generated by four identical rectangular coils wired in series, fixed to a chassis that surrounds the cell. The resulting magnetic field map has a quadrupole distribution in the transverse $x^{\prime} y$ ' plane with a zero field line lying along $z^{\prime}$. A high thermal conductivity silicone gum ensures efficient dissipation of Joule heat from the coils to the chassis. In fact under the optimal operating conditions there is a negligible temperature rise.

The cooling laser beam has a diameter of $30 \mathrm{~mm}$ to provide a large capture volume and a total power up to $200 \mathrm{~mW}$, divided by a polarising beam splitter (PBS) to supply both the $x^{\prime}$ and $y^{\prime}$ cooling directions, as shown in Fig. 1. A half-wave plate situated before the PBS is used to balance the light intensity of the two orthogonal beams, while two quarter-wave plates at the entrance of the glass cell provide the required circular polarisation. The laser is a home-made Extended Cavity Diode Laser (ECDL) centred on $852 \mathrm{~nm}$ (cesium D2 line) and injected into a Master Oscillator Power Amplifier (MOPA); it is red detuned from the transition $6 S_{1 / 2}, F=4 \rightarrow 6 P_{3 / 2}, F^{\prime}=5$ by typically $1.5 \Gamma$, where $\Gamma=2 \pi \times 5.22 \mathrm{MHz}$ is the natural width (see Fig. 3). This detuning, adjusted using the Zeeman effect in a saturation spectroscopy Cs vapour cell [26], is monitored continuously using the beat between this laser frequency and the frequency of the 3D-molasses laser (described in Subsec. 2.2). A repumper laser beam (ECDL), overlapped with the cooling beam and locked to 
$6 S_{1 / 2}, F=3 \rightarrow 6 P_{3 / 2}, F^{\prime}=4$, recovers the atoms pumped into $F=3$. It has a power of $1.5 \mathrm{~mW}$ and the same dimensions as the cooling beam, both being transmitted through the same optical fibre.

The cooling beam and the repumper cover the volume of the cuvette by total internal reflection from a series of gold-coated right-angle optical prisms, see Fig. 2b. When the beam arrives on the first prism, $42.4 \mathrm{~mm} \times 42.4 \mathrm{~mm}$ $\times 30.0 \mathrm{~mm}$ in dimensions, it is reflected successively from one perpendicular sloping face to the next before crossing the glass cell again, shifted by $30 \mathrm{~mm}$ and with opposite circular polarisation with respect to the previous passage. It is subsequently reflected by a second prism then a third, arriving finally on the fourth. The dimensions of the latter are $21.2 \mathrm{~mm} \times 21.2 \mathrm{~mm} \times 30 \mathrm{~mm}$, so the laser beam covers all its surface; here it is doubly reflected but not translated and so retraces its steps. The gold coating on the two catheti of the prisms preserves the purely $\sigma^{+}-\sigma^{-}$polarisation. A film of index matching fluid (glycerine) between the cuvette and the prisms helps minimize reflection losses at the air-glass interfaces (reduced from $4 \%$ to a negligible level) and an AR coating reduces losses at the internal faces of the cuvette. While we still measured a loss of $4 \%$ for each reflection on the gold surfaces due to the absorption, this would be reduced if we used silver-coated prisms instead [27]. Added to the cesium absorption inside the cell, these losses create an imbalance of the cooling beam intensity that might be detrimental to pre-source performance. The smallest prism was placed nearest the cell exit to minimise this effect.
The optical set-up of the pre-source is completed by another couple of laser beams, both shown in Fig. 2: a plug and a pusher. The plug beam (power $3 \mathrm{~mW}$ ) tuned to $F=4 \rightarrow F^{\prime}=5$ transition allows one to measure the longitudinal velocity distribution (see Sec. 3.4). The pusher beam (power up to $2 \mathrm{~mW}$, typical diameter $20 \mathrm{~mm}$ ) is used to make a $2 \mathrm{D}^{+}$-MOT configuration; it is tuned independently near resonance with the $F=4 \rightarrow F^{\prime}=5$ transition.

\subsection{Optical molasses and detection}

The collimated atomic beam exiting from the pre-source reaches the centre of the UHV chamber $500 \mathrm{~mm}$ downstream where we perform diagnostics using another ECDL beam that crosses the atomic beam transversely along the $y$ direction. This probe laser, scanned over the $F=4 \rightarrow$ $F^{\prime}=5$ transition, has a power up to $2 \mathrm{~mW}$ and a gaussian beam waist radius of $2.8 \mathrm{~mm}$. We select an output diameter of $5 \mathrm{~mm}$ using a diaphragm. A weak repumper laser beam $(0.2 \mathrm{~mW}$ from a Distributed Bragg Reflector diode laser, DBR) stabilised on the $F=3 \rightarrow F^{\prime}=4$ transition is overlapped with the probe laser in order to recycle atoms inevitably pumped into the $F=3$ level. Two photodetectors, one opposite the probe laser and the second in the same plane but oriented along the $x$ direction, allow simultaneous detection of the transmission and fluorescence signals.

In this region we can switch on a 3D optical molasses (3D-OM) to capture the atomic beam. This is formed by six counterpropagating beams, red detuned by $12 \mathrm{MHz}$ 
from the $F=4 \rightarrow F^{\prime}=5$ transition and circularly polarised (each pair of counterpropagating beams has opposite $\sigma^{+}-\sigma^{-}$polarisation). The horizontal retro-reflected beam ( $x$-axis) is overlapped with a weak repumper beam (power $0.1 \mathrm{~mW}$ ). The central frequency of the upward and downward pairs of beams can be modified independently using different Acoustic-Optical Modulators (AOMs) to produce either a static or a continuous moving optical molasses [13]. The beams originate from a common laser (henceforth the 3D-molasses laser), an ECDL offset-locked by $160 \mathrm{MHz}$ above the $F=4 \rightarrow F^{\prime}=4$ transition and amplified by injection into a MOPA. The output of the MOPA passes through an AOM centered on $80 \mathrm{MHz}$ to produce the desired cooling frequency. The power per beam is $7 \mathrm{~mW}$ and the waist radius $13 \mathrm{~mm}$.

\section{Main characteristics of pre-source and influence of parameters: experimental results}

In the following we present the experimental characterisation of the slow atomic beam obtained for the 2D-MOT $\left(2 \mathrm{D}^{+}-\mathrm{MOT}\right)$ and for the configuration with no $\nabla \mathrm{B}(2 \mathrm{D}-$ OM). We studied the detected atomic flux as a function of cooling laser beam power, $x^{\prime}-y^{\prime}$ power imbalance, frequency detuning and polarisation. We measured the transverse and longitudinal velocity distributions of the collimated atomic beam by Doppler broadening and Time of Flight (TOF), respectively. Finally, we analysed the transverse beam profile by translating the whole detection system perpendicularly to the atomic beam.
In this section all the results were obtained for a cesium pressure in the pre-source of $\sim 4 \times 10^{-8}$ mbar and calibrated as described in Appendix A. The influence of Cs pressure is postponed until Sec. 6 .

\subsection{Atomic beam flux density}

We have investigated the influence of various parameters on the detected atomic flux density. We collect the fluorescence emitted from the atomic beam excited by a transverse probe laser.

Fig. 4 shows the effect of cooling laser power. For the maximum value available $(\sim 200 \mathrm{~mW})$, the signal still increases though less steeply. This implies greater flux yields were more laser power available.

As for cooling laser detuning, Fig. 5 indicates an optimum value around $-1.5 \Gamma$ for the $2 \mathrm{D}-\mathrm{MOT}$ and somewhat higher for the 2D-OM $(-2 \Gamma)$. In the latter configuration no dependence upon laser polarisation is observed, i.e. essentially Doppler cooling acts with no sign of sub-Doppler cooling mechanisms.

\subsection{Transverse velocity}

To deduce the transverse velocity spread of the collimated atomic beam, we observed the transmission of a weak probe laser beam oriented along $y$ perpendicularly to the atomic beam. For an atom at rest we would obtain a resonance curve with a lorentzian shape whose FWHM were given by the $5.22 \mathrm{MHz}$ natural width. Ideally, once the slight saturation broadening is accounted for, the reso- 
nance curve would be only broadened by the transverse velocity distribution of the atomic beam. The one observed has a FWHM of at most $6.3 \mathrm{MHz}$ (see Fig. 6 for a typical result). However, some of this broadening is almost certainly attributable to slight irreproducibility of the frequency scan ramp. By fitting this curve with a Voigt profile (convolution between a gaussian velocity distribution and a lorentzian distribution with the natural width), we obtain a conservative upper limit of the full velocity spread of about $1 \mathrm{~m} \cdot \mathrm{s}^{-1}$. For a longitudinal velocity of $25 \mathrm{~m} \cdot \mathrm{s}^{-1}$ (see Sec. 3.4) this corresponds to a full beam divergence $\leq 40 \mathrm{mrad}$. We observed no significant difference between the three pre-source configurations.

\subsection{Atomic beam profile}

We measured the transverse beam density profile by translating the whole detection system (probe laser and transmission photodetector) perpendicularly to the axis of the atomic beam, with the probe transverse to the atomic beam. The probe beam diameter was reduced from 5 to $2 \mathrm{~mm}$ to provide better spatial resolution. The probe frequency was locked to the centre of the $F=4 \rightarrow F^{\prime}=5$ transition. The width (FWHM) of the spatial distribution is found to be $9 \mathrm{~mm}$ for $\nabla \mathrm{B} \simeq 1.3 \mathrm{G} / \mathrm{cm}$ (value optimising the atomic flux) and $15 \mathrm{~mm}$ for $\nabla \mathrm{B}=0$ (Fig. 7). For a point source these would correspond to a maximum full beam divergence of 18 and $30 \mathrm{mrad}$ respectively, compatible with the limit inferred from Doppler spectroscopy (see Sec. 3.2). The narrower profile obtained in the MOT configuration was expected since the Doppler cooling process operates in a tighter spatial confinement than in the OM. However, this difference in divergence translated into transverse velocity spread is too small to be distinguished by the transverse Doppler spectroscopy presented above. We conclude that the source is highly directional, a valuable attribute for efficient loading of a 3D-optical molasses.

\subsection{Longitudinal velocity}

For TOF measurements, the plug laser, modulated by a mechanical chopper wheel $(\mathrm{f}=7 \mathrm{~Hz})$, cuts the slow atomic beam into slices of $1.5 \mathrm{~ms}$ duration. For each of these, at a distance $\mathrm{L}=500 \mathrm{~mm}$ downstream, we record the fluorescence induced by the cw transverse probe beam tuned to the $F=4 \rightarrow F^{\prime}=5$ transition. The fluorescence signal is proportional to the instantaneous atomic density $n(L, t)$ from which the longitudinal velocity distribution of atomic flux

$$
\varphi(v) d v=v \cdot n(L, t) d t
$$

can be reconstructed using $v=L / t . \Phi=\int \varphi(v) d v$ is the total flux. The experimental conditions ( $\nabla \mathrm{B}$ and cooling beam detuning) were optimised to yield the maximum flux in continuous operation. Fig. 8 shows the results for the different pre-source configurations. No essential difference is observed between the 2D-OM and 2D-MOT: peak velocity around $25 \mathrm{~m} \cdot \mathrm{s}^{-1}$ and full velocity spread about $50 \mathrm{~m} \cdot \mathrm{s}^{-1}$, slightly larger for the OM case together with a reduction in total flux of $\simeq 30 \%$. However, for the $2 \mathrm{D}^{+}$MOT configuration, in addition to the distribution found 
in the 2D-MOT case, a narrow peak (FWHM $5 \mathrm{~m} \cdot \mathrm{s}^{-1}$ ) appears centred at the low velocity of $13 \mathrm{~m} \cdot \mathrm{s}^{-1}$. This structure increases the total flux, though by no more than $30 \%$. Incidentally, we find the same gain in total flux for a configuration $2 \mathrm{D}^{+}-\mathrm{OM}$ with respect to the simple $2 \mathrm{D}-\mathrm{OM}$.

We have confirmed these results by Doppler absorption spectroscopy. Due to limited optical access, we cannot align the probe along the atomic beam axis, as desired for maximum sensitivity to the longitudinal velocity. However we can align it along the $\pm x$ horizontal axis at $\pm 45^{\circ}$ to the atomic beam. The $5.22 \mathrm{MHz}$ natural width then limits the velocity resolution to $\Delta v=\sqrt{2} \lambda \cdot \Gamma / 2 \pi \simeq$ $6 \mathrm{~m} \cdot \mathrm{s}^{-1}$. With no repumper, the Doppler shifted probe absorption signal directly yields the atomic beam flux distribution $\varphi(v)$. Fig. 9 shows the results for the 2D- and $2 \mathrm{D}^{+}$-MOTs and both co- and contra-propagating probe beams. Though noisy, they compare favourably with the distributions obtained using TOF (see Fig. 8). In particular the $2 \mathrm{D}^{+}$configuration provides additional flux at lower velocities (peak around $15 \pm 2 \mathrm{~m} \cdot \mathrm{s}^{-1}$ ) compared to the 2D's (peaked around $23 \pm 2 \mathrm{~m} \cdot \mathrm{s}^{-1}$ ).

Similar tests were performed with the signal-to-noise ratio enhanced using a repumper tuned to $F=3 \rightarrow F^{\prime}=$ 4. However, care must be taken in this case to avoid velocity selective hyperfine pumping since the repumper frequency is fixed. Thus we employed a $30 \mathrm{~mm}$-dia. repumper transverse to both probe and atomic beams. Note that here one measures the atomic beam density distribution $d n / d v$ from which the flux distribution $v \cdot d n / d v$ can be recovered. The results also agree well for both the $2 \mathrm{D}$ - and $2 \mathrm{D}^{+}$-MOTs and for co- and contra-propagating probe beams.

\section{Comparison with other similar work}

As already pointed out, our device differs from other MOTs via the use of gold-coated optical prisms in place of mirrors and quarter-wave plates. Yet this novel simplification does not hinder performance and the source stands comparison with similar work. To illustrate this we discuss the traps of references $[3,19,24]$ and mention some important differences. In Tab. 1 we summarise the characteristics of the slow beams analysed in this section as well as those of references $[15,22,25]$.

- In our pre-source the cooling laser power is recycled, which allows one to work with cooling beams of at most $220 \mathrm{~mW}$. In [3] the total power is similar to ours but is split into four beams while in [24] the cooling laser light, provided by a Ti:sapphire laser, has a power of $160 \mathrm{~mW}$ per beam. The trap in [19] is a compact magneto-optical source where the total power is only $60 \mathrm{~mW}$, carefully distributed among the four beams. Indeed, a possible criticism of our design concerns the imbalance of the light intensity due to absorption losses in vapour and optics; lower losses would surely improve performance.

- We employ an optimised magnetic field gradient in the range of $1.0-1.5 \mathrm{G} / \mathrm{cm}$, a value more than an order of magnitude lower than that used in $[3,24]$ though the cell dimensions are quite similar. In [19] this dif- 
ference is less though their trap has a considerably smaller volume. A low magnetic field gradient is desirable for many applications where stray fields are harmful (atomic clocks for instance). Moreover, our source works almost as well with no gradient at all, which is even better from this point of view. This behaviour we attribute to the large size of the exit hole $(16 \mathrm{~mm})$ which allows cold atoms to contribute to the slow beam be they close to the axis or not.

- Our measured atomic flux, even if obtained at the lower vapour pressure in the pre-source of $\sim 4 \times 10^{-8}$ mbar is twice higher than that of [19] and of the same order as the one in [3]. In [24] the atomic flux is 4 times higher than ours for a vapour pressure in their cell set to the optimum value of $2 \times 10^{-6}$ mbar though longterm contamination by Cs might be an issue at such high density.

- Finally, the longitudinal velocity of the atomic beam measured in this work has a broad distribution centred around $27 \mathrm{~m} \cdot \mathrm{s}^{-1}$ for the $2 \mathrm{D}-\mathrm{MOT}$ configuration, a value higher than those of [3] $\left(20 \mathrm{~m} \cdot \mathrm{s}^{-1}\right)$ and [19] $\left(\sim 10 \mathrm{~m} \cdot \mathrm{s}^{-1}\right)$. A low longitudinal velocity would be an advantage for Doppler capture and in fact for our $2 \mathrm{D}^{+}$MOT configuration, the longitudinal velocity distribution is reminiscent of that described in [24], with a narrow peak around $15 \mathrm{~m} \cdot \mathrm{s}^{-1}$, ideal for loading a $3 \mathrm{D}$ molasses, as discussed below.

\section{Capturing the atomic beam in the static 3D optical molasses}

We have built the pre-source described above to improve the useful atomic flux in a continuous cesium fountain clock [10]. This clock is currently fed by loading a 3D-OM from a thermal cesium vapour. In this section we analyse the gain obtainable by loading a 3D-OM by the slow atomic beam provided by the pre-source (see Fig. 13 for the experimental schematic).

For this, we measured the probe absorption with the 3D-molasses laser turned on. In a preliminary step, the optimum detuning of this laser for maximum capture was determined to be around $-12 \mathrm{MHz}$ whatever the loading technique (Fig. 10). Then the parameters of the pre-source were adjusted to optimise the absorption signal: we found them to be slightly different (cooling laser detuning $\delta \simeq$ $11 \mathrm{MHz}$ and $\nabla \mathrm{B} \simeq 0.8 \mathrm{G} / \mathrm{cm}$ ) than without capture. When we switched from the $2 \mathrm{D}$ - to the $2 \mathrm{D}^{+}$-MOT configuration, most remarkably, the absorption signal was increased by a factor of $\simeq 1.8$ while without capture the gain was only $30 \%$ (see Fig. 8). A similar behaviour was observed for a $2 \mathrm{D}$ - versus $2 \mathrm{D}^{+}{ }_{-} \mathrm{OM}$ comparison. These effects we ascribe to increased 3D-OM capture efficiency for slow atoms.

Next we compare probe absorption for the 3D-OM loading by a thermal cesium vapour or by the slow atomic beam. For this purpose we use a second reservoir and isolate the pre-source from the UHV chamber by closing the gate valve. Typical results are shown in Fig. 11. 
Table 1. Summary of the characteristics of various slow beam sources. $\nabla \mathbf{B}$ is the magnetic field gradient, $v_{L}$ the peak longitudinal velocity and $P_{\text {tot }}$ the cooling laser total power.

\begin{tabular}{|c|c|c|c|c|c|c|c|}
\hline Parameter & $\begin{array}{l}\text { Present } \\
\text { Work }^{a}\end{array}$ & $\begin{array}{c}\text { Chelkowski } \\
\text { Ref. }[3]^{b}\end{array}$ & $\begin{array}{c}\text { Kohel et al. } \\
\text { Ref. }[15]^{c}\end{array}$ & $\begin{array}{l}\text { Ovchinnikov } \\
\text { Ref. }[19]^{a}\end{array}$ & $\begin{array}{l}\text { Berthoud et al. } \\
\text { Ref. }[22]^{d}\end{array}$ & $\begin{array}{l}\text { Schoser et al. } \\
\text { Re. }[24]^{a}\end{array}$ & $\begin{array}{c}\text { Dieckmann et al. } \\
\text { Ref. }[25]^{a}\end{array}$ \\
\hline Flux $\left[10^{9} \mathrm{at} \cdot \mathrm{s}^{-1}\right]$ & 13 & 11 & 2.2 & 8 & 0.13 & 60 & 9 \\
\hline Pressure $\left[10^{-8}\right.$ mbar $]$ & 4 & n.a. & 0.25 & 32 & 4 & 160 & 15 \\
\hline$\nabla \mathbf{B}\left[\mathrm{G} \cdot \mathrm{cm}^{-1}\right]$ & $1.0-1.5$ & 19 & n.a. & 6 & 12.5 & 17 & 12.6 \\
\hline$v_{L}\left[\mathrm{~m} \cdot \mathrm{s}^{-1}\right]$ & 20 & 20 & 15 & 11 & $0.7-3$ & 15 & 8 \\
\hline$P_{t o t}[\mathrm{~mW}]$ & 220 & 220 & 600 & 60 & 60 & 640 & 34 \\
\hline
\end{tabular}

${ }^{a} 2 \mathrm{D}^{+}$-MOT, ${ }^{b}$ 2D-MOT, ${ }^{c}$ Pyramidal trap, ${ }^{d}$ 2D-MOT+3D-OM.

For loading from a vapour $\sim 10^{-8}$ mbar we observed a Doppler broadened absorption of the order of $10-15 \%$ along $250 \mathrm{~mm}$ of vapour column (internal diameter of the chamber) with small sub-Doppler peaks centered on the $F=4 \rightarrow F^{\prime}=4$ and $F=4 \rightarrow F^{\prime}=5$ atomic resonances due to slow atoms captured in the 3D-OM. These have to be compared with the peaks associated with slow beam loading: the capture efficiency is $\sim 8$ times higher in the latter case. A second positive aspect provided by using the pre-source is highlighted by the images recorded by a CCD camera (Fig. 12). The striking difference is the presence of fluorescent light along the 3D-molasses laser beam axes in Fig. 12b, indicating diffuse vapour absent in Fig. 12a. This confirms the confinement of the atoms in the region of interest and quasi-absence $\left(4 \times 10^{-10} \mathrm{mbar}\right)$ of thermal background atoms for pre-source loading the 3D-OM despite the large exit hole and the absence of getters.
Finally, we increased the useful atomic flux still further by raising the cesium pressure inside the pre-source (kept constant until now at about $4 \times 10^{-8}$ mbar). This aspect is analysed in the next section, where we deal with the launch of the slow atoms captured by the 3D-OM in a vertical fountain.

\section{Launch configuration for the optical}

\section{molasses}

The slow atoms captured by the 3D-OM are launched vertically using a moving optical molasses. The set-up is shown in Fig. 13. Here the two up-going (down-going) beams are detuned by $+3 \mathrm{MHz}(-3 \mathrm{MHz})$ with respect to the horizontal retro-reflected beam, held at $-12 \mathrm{MHz}$ from the $F=4 \rightarrow F^{\prime}=5$ transition. This frequency difference provides a vertical launch velocity of $3.6 \mathrm{~m} \cdot \mathrm{s}^{-1}$. The fountain beam was detected $470 \mathrm{~mm}$ above the 3DOM using both fluorescence and absorption induced by 
Table 2. Summary of methods for loading a continuous cold atom fountain. Atomic flux density $\Phi$ and transverse diameter $\varnothing$ of fountain beam at a distance of $470 \mathrm{~mm}$ above the $3 \mathrm{D}-\mathrm{OM}$ source, with a vertical launch velocity of $3.6 \mathrm{~m} \cdot \mathrm{s}^{-1}$. Comparison for pre-source (2D- and $\left.2 \mathrm{D}^{+}-\mathrm{MOT}\right)$ at Cs vapour pressure $1.2 \times 10^{-7}$ mbar and for vapour loading at $0.8 \times 10^{-8}$ mbar.

\begin{tabular}{lccc}
\hline & Vapour & 2D-MOT & $2 \mathrm{D}^{+}$-MOT \\
\hline$\Phi\left(10^{13} \mathrm{at} \cdot \mathrm{s}^{-1} \cdot \mathrm{m}^{-2}\right)$ & 0.4 & 4.5 & 8.5 \\
\hline$\varnothing(\mathrm{mm})$ & $16.4(0.3)$ & $15.0(0.1)$ & - \\
\hline
\end{tabular}

the probe laser beam ( 0.5 to $2 \mathrm{~mW}$ power, $5 \mathrm{~mm}$ diameter) for different loading techniques of the source.

Fig. 14 shows the main result of this work, namely that 2D-MOT-slow-beam loading gives 10 times more flux than vapour capture; the $2 D^{+}-$MOT almost doubles this. The transverse cold beam profiles also recorded for the 2D-MOT- and vapour-loading of the 3D-OM were quite similar. Tab. 2 summarises these results.

As regards the impact of the cesium pressure inside the pre-source (Fig. 15), we find a maximum atomic flux $\left(\Phi=8.5 \times 10^{13}\right.$ at $\left.\cdot \mathrm{s}^{-1} \cdot \mathrm{m}^{-2}\right)$ for the rather high value of $1.2 \times 10^{-7}$ mbar, corresponding to an absorption of $17 \%$ in the pre-source (for a $3 \mathrm{~cm}$ transverse path). While this is encouraging, in practice a somewhat lower pressure might be used to extend the working lifetime of the device. For even higher pressure, the detected flux falls due to the greater collision rate and increased imbalance of the cooling laser beams due to higher absorption in the pre-source.

\section{Conclusions and perspectives}

We have developed and completely characterised a twodimensional source of slow Cs atoms with the aim of loading a continuous atomic beam fountain. A comparison of the characteristics for the $2 \mathrm{D}-\mathrm{MOT}, 2 \mathrm{D}-\mathrm{OM}$ and $2 \mathrm{D}^{+}$MOT configurations is summarised in Tab. 3. The innovative set-up with $i$ ) low cost optics, $i i$ ) absence of differential pumping and iii) exit hole with no diaphragm, is shown not to limit the performance of our device. The slow atomic beam provided by the pre-source has been captured in a UHV chamber by a 3D-OM 500 mm downstream and then launched in a vertical flight. Loading the molasses using the 2D-MOT provides a gain in the detected flux by a factor of $\simeq 10$ compared with thermal cesium vapour capture, the method used heretofore to feed our atomic clock fountain. An added advantage is the quasi-absence of thermal background atoms. The introduction of a pusher beam in the pre-source $\left(2 \mathrm{D}^{+}\right.$MOT) increases the fountain flux by a further factor of $\simeq 1.8$, as a result of a more favorable longitudinal velocity distribution. This suggests we might boost the fountain flux even more by shortening the length of the pre-source cell, as demonstrated in [24]. At the optimum vapour pressure in the pre-source of $1.2 \times 10^{-7} \mathrm{mbar}$ and for a $2 \mathrm{D}^{+}$MOT configuration we achieved a highest flux density of $8.5 \times 10^{13}$ at $\cdot \mathrm{s}^{-1} \cdot \mathrm{m}^{-2}$. Our results imply a potential gain of $\sqrt{20}$ in stability of an atomic shot noise limited fountain clock, though the signal-to-noise remains to be investigated. 
Table 3. Comparison of the characteristics of the slow atomic beam for the 2D-MOT, 2D-OM and 2D ${ }^{+}$-MOT configurations. $\Phi$ is the maximum atomic flux density, $v_{L}$ the peak longitudinal velocity and $\Delta \mathrm{v}_{T}$ the transverse velocity spread. The total flux is calculated using the transverse beam size measured at $500 \mathrm{~mm}$ from the exit (see fig. 7) and assuming it to be the same for the $2 \mathrm{D}$ - and the $2 \mathrm{D}^{+}$-MOT configurations. Note that Cs vapour pressure in the pre-source is $\sim 4 \times 10^{-8}$ mbar, namely one third of the optimum value for fountain flux measurements, see Tab. 2 and Fig. 15.

\begin{tabular}{lccc}
\hline & 2D-MOT & 2D-OM & $2 \mathrm{D}^{+}-\mathrm{MOT}$ \\
\hline$\Phi\left(10^{14} \mathrm{at} \cdot \mathrm{s}^{-1} \cdot \mathrm{m}^{-2}\right)$ & 1.0 & 0.7 & 1.3 \\
$v_{L}\left(\mathrm{~m} \cdot \mathrm{s}^{-1}\right)$ & 27 & 25 & $20^{*}$ \\
$\Delta_{\mathrm{v}}\left(\mathrm{m} \cdot \mathrm{s}^{-1}\right)$ & $\leq 1$ & $\leq 1$ & $\leq 1$ \\
Total flux $\left(10^{10} \mathrm{at} \cdot \mathrm{s}^{-1}\right)$ & 1 & 1 & 1.3 \\
\hline
\end{tabular}

\footnotetext{
* This value is found by averaging the two peaks that characterise the longitudinal velocity distribution for the $2 \mathrm{D}^{+}$-MOT configuration (Fig. 8).
}

This work was funded by the Swiss National Science Foundation and the Swiss Federal Office of Metrology and Accreditation (METAS). The authors acknowledge helpful discussions with F. Fuso and Y.B. Ovchinnikov and thank C. Affolderbach for his kind reading of the manuscript. The Observatoire cantonal thanks the CNRS for making possible the stay of J.G. in Neuchâtel.

\section{A Appendix: Flux calibration}

We have calibrated the atomic flux using probe absorption without repumper rather than fluorescence signals to be independent of light collection efficiency. We exploit the two $F=4 \rightarrow F^{\prime}=4$ and $F=4 \rightarrow F^{\prime}=5$ atomic resonances. Strictly we detect only atoms present in the $F=4$ ground hyperfine level. On the former open transition, absorption is limited by the hyperfine pumping time with an absolute value independent of probe beam intensity and size. The power subtracted from the beam yields directly the atomic flux. This method is the most reliable but gives small signals. For this reason, we also use the $F=4 \rightarrow F^{\prime}=5$ transition as a transfer standard since it provides better $\mathrm{S} / \mathrm{N}$. On this closed (cycling) transition the absorption is limited by the transit time and the relative absorption gives access to the atomic density. We have also exploited it to cross-check the 44' calibration.

\section{A.1 Absorption on the $F=4 \rightarrow F^{\prime}=4$ component (pumping transition)}

Atoms excited by the probe beam to the $6 P_{3 / 2}, F^{\prime}=4$ level undergo spontaneous emission that puts them in either the $F=3$ or $F=4$ ground state, with a probability given by the branching ratio (Tab. 4). Since there is no repumper, $12 / 5$ photons on average are emitted per atom before it is pumped into $F=3$ and lost. The corresponding emitted power equals the absorbed probe power. From the absolute amplitude of the absorption peak $S_{a b s}$ one can deduce this power $P$, given the photodetector transimpedance $R_{f}$ and quantum efficiency $\eta_{P D}$, and the transmission of the detection optics $\eta_{O p}$. This power divided by the energy removed per atom yields the atomic 
Table 4. Branching ratio in spontaneous emission $a_{e^{\prime} g}$ between an excited state $e^{\prime}$ and a ground state $g$ for the ${ }^{133} \mathrm{Cs}$ D2 line.

\begin{tabular}{ccccc}
\hline$a_{e^{\prime} g}$ & $F^{\prime}=2$ & $F^{\prime}=3$ & $F^{\prime}=4$ & $F^{\prime}=5$ \\
\hline $\mathrm{F}=3$ & 1 & $3 / 4$ & $5 / 12$ & 0 \\
$\mathrm{~F}=4$ & 0 & $1 / 4$ & $7 / 12$ & 1 \\
\hline
\end{tabular}

flux traversing the probe beam and thereby the atomic flux density

$$
\Phi=\frac{S_{a b s}}{R_{f} \eta_{P D} \eta_{O p}(12 / 5 h \nu) \Sigma_{d e t}}
$$

where $\Sigma_{\text {det }}=$ probe diameter $\times$ the transverse size of the atomic beam is the detection area. With $S_{a b s}=1(0.3) \mathrm{mV}$ (see Fig. 16), $R_{f}=1 \mathrm{M} \Omega, \eta_{P D}=0.5 \mathrm{~A} / \mathrm{W}, \eta_{O p}=0.5$ and $\Sigma_{\text {det }}=5 \times 10 \mathrm{~mm}^{2}$, we obtain

$$
\Phi_{44^{\prime}}=1.5(0.5) \times 10^{14} \mathrm{at} \cdot \mathrm{s}^{-1} \cdot \mathrm{m}^{-2} .
$$

The uncertainty here is mainly statistical.

\section{A.2 Absorption on the $F=4 \rightarrow F^{\prime}=5$ component (cycling transition)}

In the limit of negligible optical saturation and negligible off-resonant hyperfine pumping, the resonant probe relative absorption $\Delta I / I$ by the atomic beam of column length $\Delta y$ gives the atomic density $n$ (using for instance Ref. [8]). Multiplying by the mean longitudinal velocity $v$, one obtains the flux density :

$$
\Phi=n v=-\frac{27}{11} \frac{\Delta I}{I} \frac{1}{\sigma} \frac{v}{\Delta y} .
$$

Table 5. Relative transition strengths in absorption $w_{g e^{\prime}}$ and in stimulated emission $w_{e^{\prime} g}$ between a ground state $g$ and an excited state $e^{\prime}$ for the ${ }^{133}$ Cs D2 line.

\begin{tabular}{ccccc}
\hline$w_{g e^{\prime}}=w_{e^{\prime} g}$ & $F^{\prime}=2$ & $F^{\prime}=3$ & $F^{\prime}=4$ & $F^{\prime}=5$ \\
\hline $\mathrm{F}=3$ & $5 / 21$ & $1 / 4$ & $5 / 28$ & 0 \\
$\mathrm{~F}=4$ & 0 & $7 / 108$ & $7 / 36$ & $11 / 27$ \\
\hline
\end{tabular}

Here $\sigma=3 \lambda^{2} / 2 \pi$ is the resonant cross section and $11 / 27$ the relative oscillator strength for the $F=4 \rightarrow$ $F^{\prime}=5$ transition, see Tab. 5 . For $\Delta I / I=0.23 \%$ (Fig. 16), $v=25 \mathrm{~m} \cdot \mathrm{s}^{-1}, \Delta y=10 \mathrm{~mm}$ (atomic beam transverse size), we obtain

$$
\Phi_{45^{\prime}}=0.5 \times 10^{14} \mathrm{at} \cdot \mathrm{s}^{-1} \cdot \mathrm{m}^{-2} .
$$

The difference between the results of the two calibration methods we attribute to the difficulty of estimating all the uncertainties in the factors involved in the 45' calibration (e.g. Zeeman pumping, optical saturation, beam geometry, velocity spread, etc.). We consider the 44' calibration to be more reliable and thus place a conservative lower limit of $1.0 \times 10^{14} \mathrm{at} \cdot \mathrm{s}^{-1} \cdot \mathrm{m}^{-2}$ on the atomic flux density for the slow beam provided by the pre-source in the 2D-MOT configuration and for a cesium pressure of $\simeq 4 \times 10^{-8} \mathrm{mbar}$

\section{A.3 Fluorescence}

Many signals presented in this article show fluorescence. They were calibrated by simultaneous recording of absorption signals characterised above. 


\section{References}

1. P.R. Berman, Atom Interferometry (Academic Press, San Diego, 1997).

2. J. Stuhler, M. Fattori, T. Petelski and G.M. Tino, J. Opt. B: Quantum Semiclass. Opt. 5, S75-S81 (2003).

3. S. Chelkowski, Diplomarbeit, Institut of Quantum Physics, University of Hannover (2002). Unpublished.

4. S. Sanguinetti, J. Guéna, M. Lintz, Ph. Jacquier, A. Wasan and M.-A. Bouchiat, Eur. Phys. J. D 25, 3-13 (2003).

5. V. Natarajan, Eur. Phys. J. D 32, 33-38 (2005).

6. R. Wynands and S. Weyers, Metrologia 42, S64-S79 (2005).

7. J. Vanier, Appl. Phys. B 81, 421-442 (2005).

8. H.J. Metcalf and P. van der Straten, Laser Cooling and Trapping (Springer-Verlag, New York, 1999).

9. A. Grabowski, R. Heidemann, R. Löw, J. Stuhler and T. Pfau, arXiv:quant-ph/0508082 v1 (2005).

10. G. Dudle, A. Joyet, N. Castagna, G. Mileti, P. Thomann, C. Mandache and T. Acsente, in Proceedings of the 16th EFTF, St. Petersburg, 12-14 March 2002 (St. Petersburg State University of Aerospace Instrumentation, Russia), E014-E-016 (2002).

11. A. Joyet, G. Mileti, P. Thomann and G. Dudle, IEEE Trans. Instr. Meas. 50, 150-156 (2001).

12. A. Composeo, A. Piombini, F. Cervelli, F. Tantussi, F. Fuso and E. Arimondo, Opt. Commun. 200, 231-239 (2001)

13. P. Berthoud, E. Fretel and P. Thomann, Phys. Rev. A 60, R4241-R4244 (1999).

14. M.A. Joffe, W. Ketterle, A. Martin and D.E. Pritchard,
15. J.M. Kohel, J. Ramirez-Serrano, R.J. Thompson, L. Maleki, J.L. Bliss and K.G. Libbrecht, J. Opt. Soc. Am. B 20, 1161-1168 (2003).

16. L. Cacciapuoti, A. Castrillo, M. de Angelis and G.M. Tino, Eur. Phys. J. D 15, 245-249 (2001).

17. H. Wang and W.F. Buell, J. Opt. Soc. Am. B 20, 20252030 (2003).

18. R.N. Watts and C.E. Wieman, Opt. Lett. 11, 291-293 (1986).

19. Y.B. Ovchinnikov, Opt. Commun. 249, 473-481 (2005).

20. Z.T. Lu, K.L. Corvin, M.J. Renn, M.H. Anderson, E.A. Cornell and C.E. Wieman, Phys. Rev Lett. 77, 33313334 (1996).

21. H. Chen and E. Riis, Appl. Phys. B 70, 665-670 (2000).

22. P. Berthoud, A. Joyet, G. Dudle, N. Sagna and P. Thomann, Europhys. Lett. 41, 141-146 (1998).

23. P. Cren, C.F. Roos, A. Aclan, J. Dalibard and D. GuéryOdelin, Eur. Phys. J. D 20, 107-116 (2002).

24. J. Schoser, A. Batär, R. Löw, V. Schweikhard, A. Grabowski, Y.B. Ovchinnikov and T. Pfau, Phys. Rev. A 66, 023410-1 - 023410-10 (2002).

25. K. Dieckmann, R.J.C. Spreeuw, M. Weidemüller and J.T.M. Walraven, Phys. Rev. A 58, 3891-3895 (1998).

26. S. Lecomte, E. Fretel, G. Mileti and P. Thomann, Appl. Opt. 39, 1426-1429 (2000).

27. C.W. Goodwin, private communication. Calculations for a $45^{\circ}$ angle of incidence and $\lambda=852 \mathrm{~nm}$ give $\mathrm{R}_{P}=96.66 \%$ and $\mathrm{R}_{S}=97.45 \%$ for $\mathrm{Au}$ and $\mathrm{R}_{P}=98.45 \%$ and $\mathrm{R}_{S}=99.21 \%$ for $\mathrm{Ag}$.

J. Opt. Soc. Am. B 10, 2257-2262 (1993). 


\section{B Figure captions}

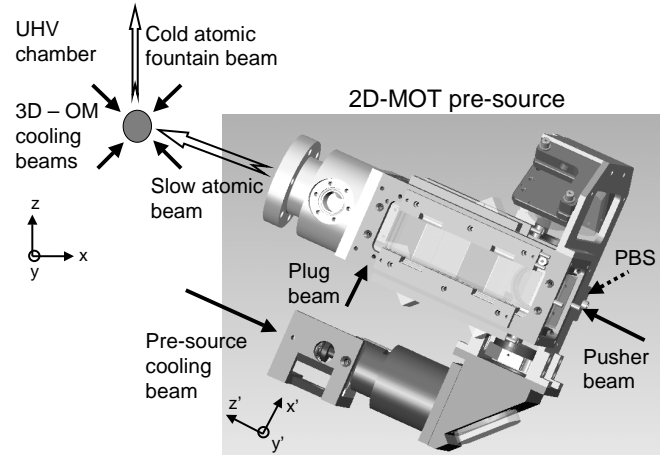

Fig. 1. Pre-source in an overview of the experimental set-up.

The cooling beam is split into two parts by a polarising beam splitter (PBS). Each then propagates along the glass cell, glued to a titanium flange, itself connected to the UHV chamber.

a)
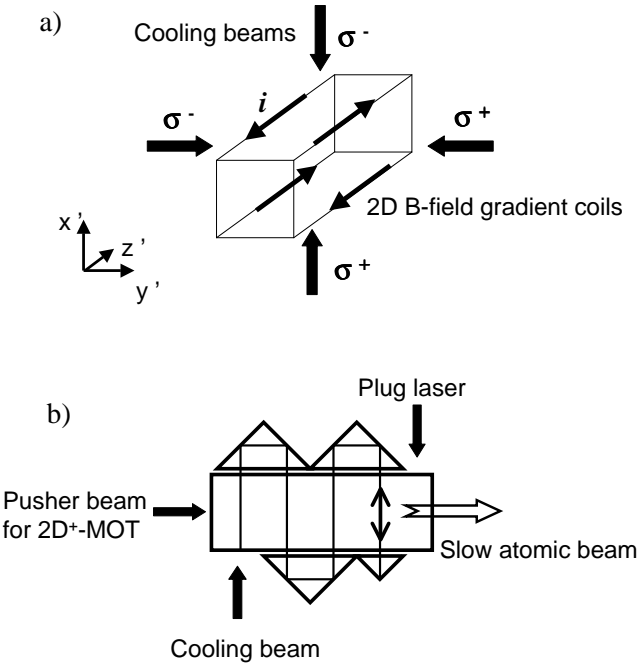

Fig. 2. Scheme of our pre-source: a) The four perpendicular cooling beams and the coils that generate the transverse magnetic field gradient. b) Beam geometry (for clarity only one of the two cooling directions is represented), see Sec. 2.1 for details.

\section{Cs atomic levels: D2 line}

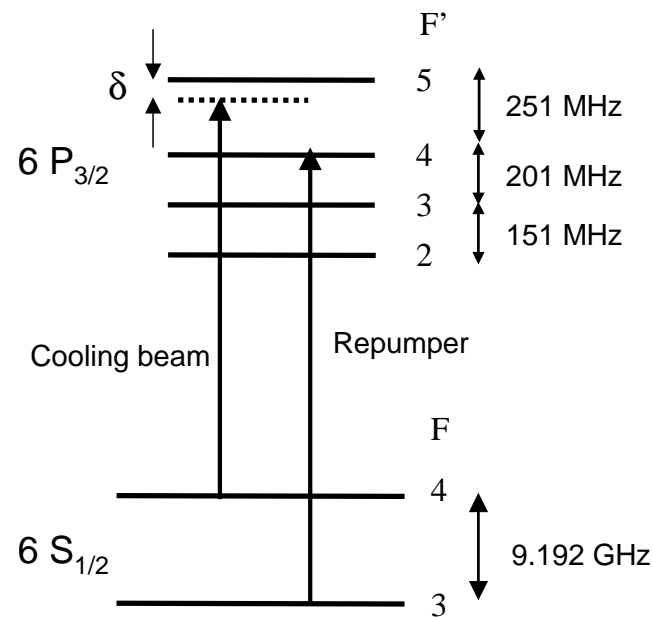

Fig. 3. Hyperfine transitions of the ${ }^{133}$ Cs D2 line (852 nm) used for the pre-source and diagnosis. The cooling beam is red detuned with respect to the $F=4 \rightarrow F^{\prime}=5$ transition by $\delta$, typically $-8 \mathrm{MHz}$.

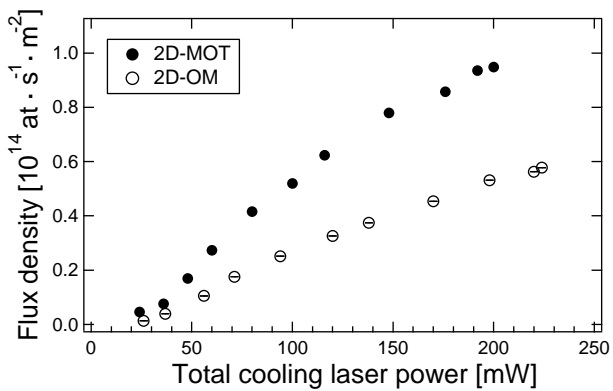

Fig. 4. Atomic flux density versus total cooling laser power measured $500 \mathrm{~mm}$ from the exit of the pre-source. The data points were obtained observing the fluorescence induced by the probe laser beam transverse to the atomic beam with a weak repumper overlapped. The cooling beam detuning from the $F=4 \rightarrow F^{\prime}=5$ transition is $-8 \mathrm{MHz}$ for both 2D-MOT and 2D-OM configurations. $\nabla \mathrm{B}=2.6 \mathrm{G} / \mathrm{cm}$ for the MOT. 


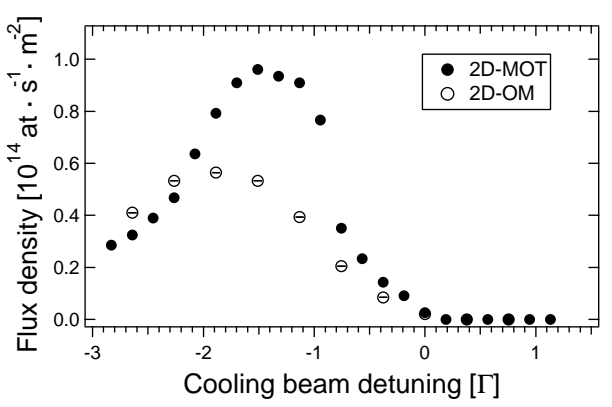

Fig. 5. Atomic flux density versus cooling laser detuning. The cooling laser power is $200 \mathrm{~mW}$. Other experimental parameters as in Fig. 4.

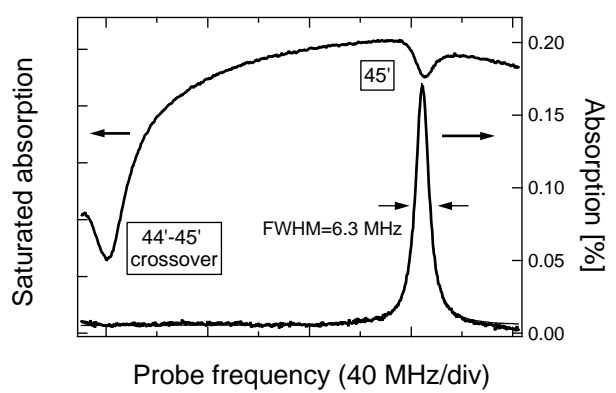

Fig. 6. Absorption spectrum of a probe laser beam transverse to the atomic beam $500 \mathrm{~mm}$ downstream. Probe saturation parameter of 0.2 . The width of the resonance gives us information about the transverse velocity spread. Upper trace: saturated absorption signal providing frequency calibration.

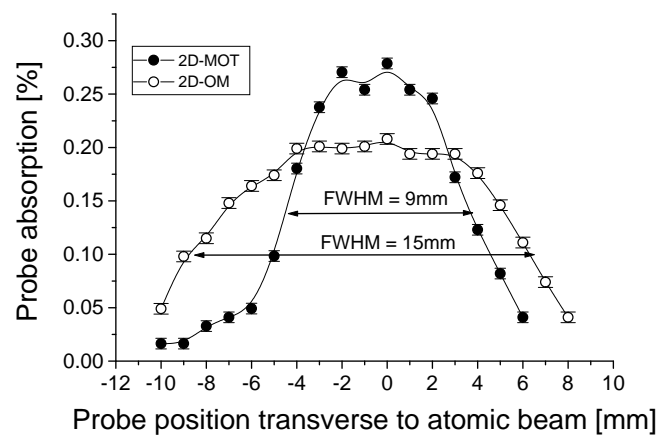

Fig. 7. Atomic beam profiles measured by probe absorption at a distance of $500 \mathrm{~mm}$ from the exit of the pre-source. Circles: standard 2D-MOT configuration; triangles: configuration with no magnetic field gradient (2D-OM); the solid lines are guidelines.

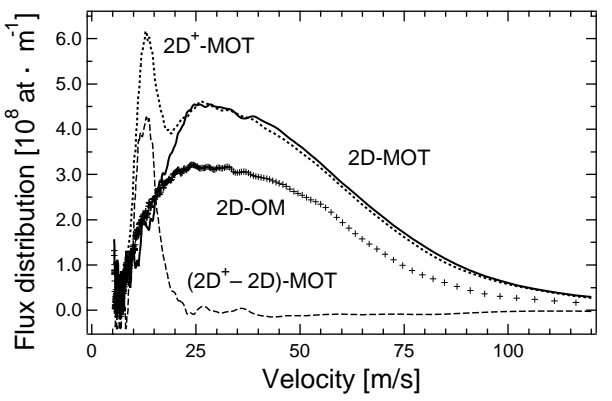

Fig. 8. Flux distribution $\varphi(v)$ as a function of the longitudinal velocity $v$ for the three configurations of the pre-source. Crosses: 2D-OM, solid line: 2D-MOT, dots: 2D ${ }^{+}-\mathrm{MOT}$ and dashed line: difference between the $2 \mathrm{D}^{+}$- and 2D-MOT's. Cooling laser detuning $-10 \mathrm{MHz}, \nabla \mathrm{B} \simeq 1.3 \mathrm{G} / \mathrm{cm}$ for the MOT's. The probe laser beam is tuned to the $F=4 \rightarrow F^{\prime}=5$ transition with the repumper overlapped. 
a)

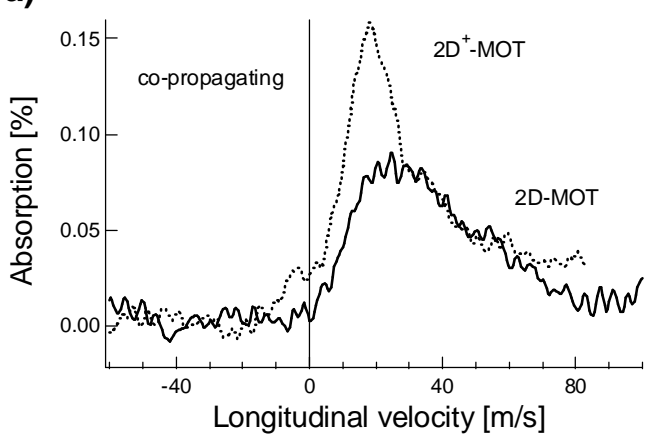

b)

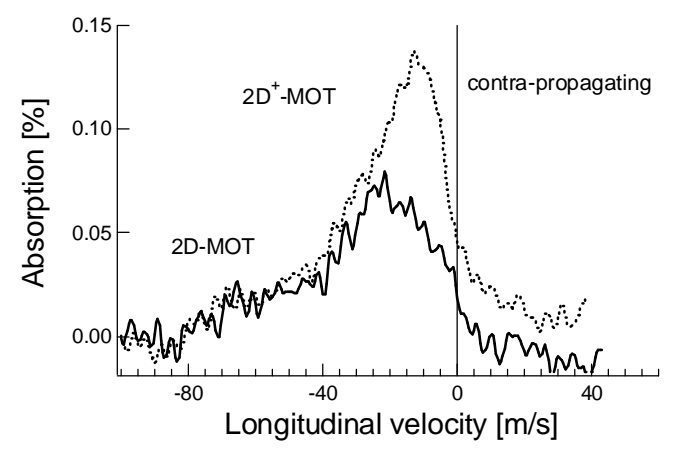

Fig. 9. Doppler shifted absorption spectra in the region of the $F=4 \rightarrow F^{\prime}=5$ transition for a probe beam oriented at $\pm 45^{\circ}$ to the atomic beam ( $\mathrm{a}$ and $\mathrm{b}$ for co- and contra-propagating geometries), without repumper. Saturating power $\simeq 2 \mathrm{~mW}$, diameter $5 \mathrm{~mm}$. The scanned probe frequency is calibrated in atomic beam longitudinal velocity $(\Delta v=\sqrt{2} \lambda \cdot \Delta \nu)$. Dotted and full lines for $2 \mathrm{D}^{+}$- and 2D-MOT pre-source.

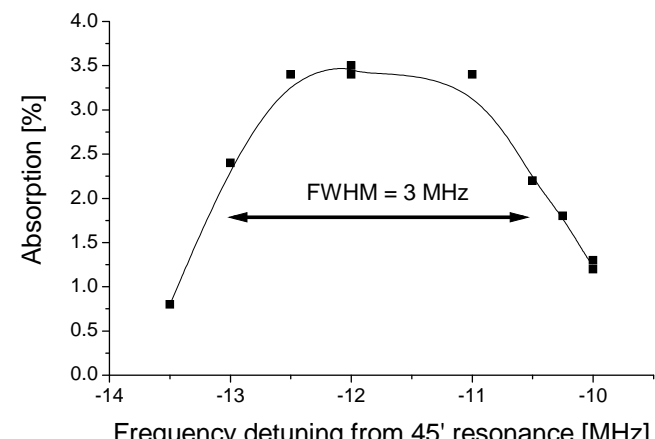

Fig. 10. Probe absorption by the 3D-optical molasses loaded by the slow atomic beam: dependence on the molasses laser detuning from the $F=4 \rightarrow F^{\prime}=5$ transition. Probe laser tuned to the $F=4 \rightarrow F^{\prime}=5$ transition.

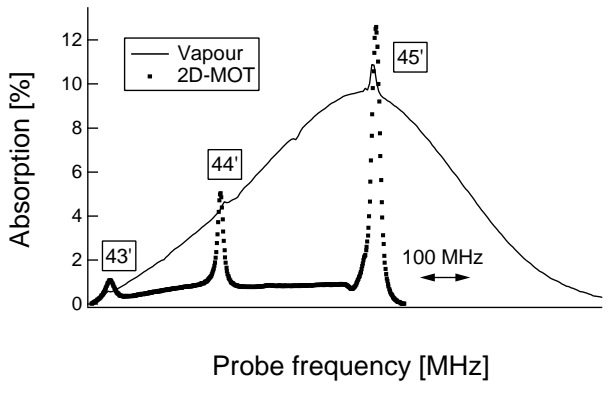

Fig. 11. Spectrum of the probe absorption across D2 line in the 3D-optical molasses region. Loading by cesium vapour (solid line) or from the 2D-MOT slow atomic beam (dotted line). Probe laser beam (diameter $5 \mathrm{~mm}$ and total power $0.15 \mathrm{~mW}$ ) overlapped by a weak repumper beam for vapour loading.

a)

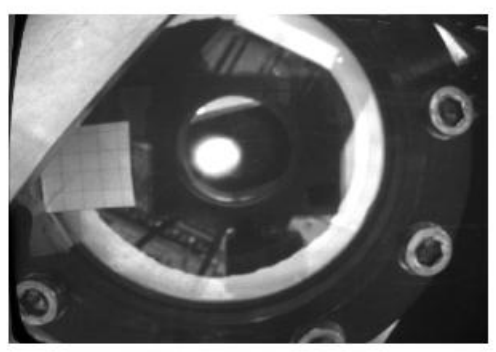

b)

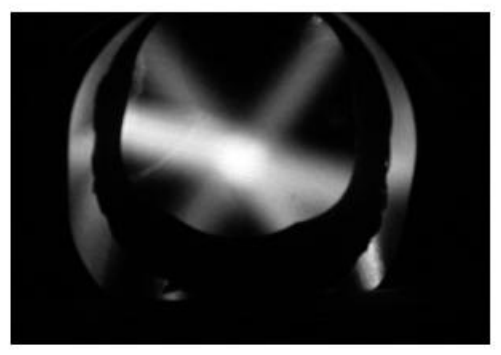

Fig. 12. Pictures showing the fluorescent light emitted in the 3D static optical molasses. a) Loading by the slow atomic beam: bright central spot of $\sim 15 \mathrm{~mm}$ diameter. Note the absence of fluorescence from the six molasses beams. b) Loading by a Cs vapour: the fluorescence induced by thermal vapour absent in a) is conspicuous along the 3D-molasses laser beam axes. 

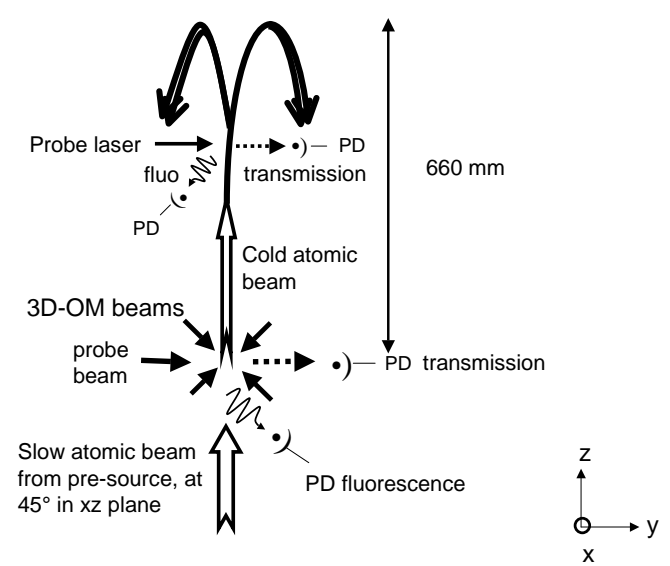

Fig. 13. Schematic of the launch configuration for the fountain. The 3D optical molasses is formed by six counterpropagating laser beams (the horizontal retro-reflected beam along $x$ is not shown) with opposite circular polarisation capture the cesium atoms travelling through the intersection region. Measurements presented in Sec. 5 are obtained with a static 3D-OM, while for those in Sec. 6 the optical molasses is in a moving configuration. Two photodetectors (PD) allow simultaneous monitoring of the fluorescence and transmission signals induced by the probe beam.

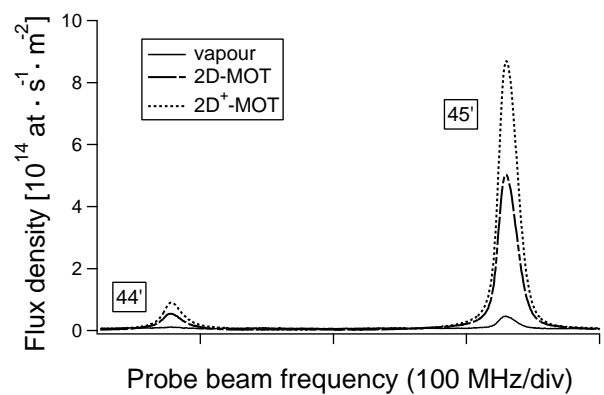

Fig. 14. Fluorescence spectrum calibrated in flux density detected $470 \mathrm{~mm}$ above the $3 \mathrm{D}-\mathrm{OM}$ source in a launch configuration upwards. Comparison between the two different techniques to load the source: from cesium vapour (full line) or from the slow atomic beam produced by the pre-source in either the 2D-MOT (dashed line) or the $2 \mathrm{D}^{+}$-MOT (dotted line) configuration. Vapour pressure $\simeq 1.2 \times 10^{-7}$ mbar in the pre-source and $\simeq 10^{-8}$ mbar in the UHV chamber.

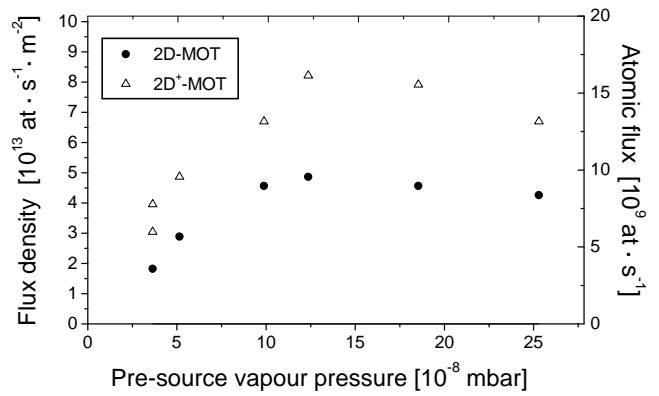

Fig. 15. Atomic flux launched vertically from a 3D moving molasses as a function of the cesium vapour pressure inside the pre-source used to load it, for both the 2D-MOT and the $2 D^{+}$-MOT configurations. Detection by induced fluorescence with probe beam tuned to the $F=4 \rightarrow F^{\prime}=5$ transition. 


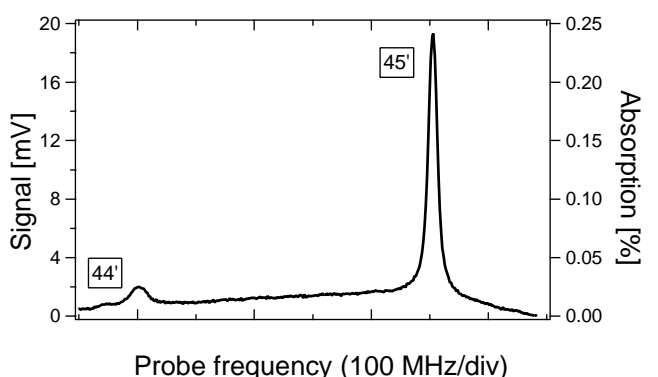

Fig. 16. Probe absorption by the slow atomic beam for low intensity light (total power $0.15 \mathrm{~mW}$, diameter $5 \mathrm{~mm}$ ) near the atomic resonances $F=4 \rightarrow F^{\prime}=4$ and $F=4 \rightarrow F^{\prime}=5$ without repumper. Here, the pre-source is a $2 \mathrm{D}-\mathrm{MOT}$ with a cesium pressure of $\simeq 4 \times 10^{-8}$ mbar. 